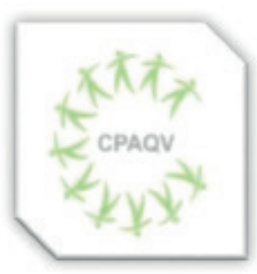

ISSN: 2178-7514
ARTIGO ORIGINAL

\section{A PREVALÊNCIA DE HIPERTENSÃO EM ADULTOS JOVENS NA ESTRATÉGIA DE SAÚDE DA FAMÍLIA MUCAJÁ}

\author{
IThe prevalence of hypertension in young adults in the mucajá family health strategy \\ Eduardo Augusto Silva Monteiro ${ }^{1}$, Leandro Lourenço Silva Monteiro ${ }^{1}$, Kaique Furtado Pureza Oliveira ${ }^{1}$, \\ Thalita da Rocha Bastos ${ }^{1}$, Tainah Lacerda Santos ${ }^{2}$, Iris Do Socorro dos Santos Marinho ${ }^{3}$, \\ Gilvanildo de Oliveira Sampaio ${ }^{3}$, Lessandra Barros Moraes Taveira ${ }^{3}$, Ednei Lopes Teixeira Marialva ${ }^{3}$, \\ Brenno Lemos da Costa ${ }^{4}$, Patricia Farias de Souza ${ }^{5}$, Tereza Cristina dos Reis Ferreira ${ }^{6}$
}

\title{
RESUMO
}

INTRODUÇ̃̃O: A Hipertensão Arterial Sistêmica (HAS) causa diversas comorbidades cardiovasculares, que estão relacionadas com piores prognósticos e risco de morte aumentado. Foram implementados no Brasil programas como o HIPERDIA, que executam o rastreamento, acompanhamento e tratamento de pacientes com HAS e Diabetes Mellitus, a fim de reduzir seu impacto na saúde pública. Então, faz-se necessário conhecer o perfil dos pacientes registrados neste programa. OBJETIVOS: Identificar a prevalência de HAS nos adultos na ESF Mucajá, criando um perfil epidemiológico destes pacientes hipertensos. MÉTODOS: Estudo retrospectivo, no qual foi feita a coleta de dados como sexo, idade, IMC, tabagismo e alcoolismo de prontuários dos pacientes da ESF Mucajá que estivessem na faixa etária determinada (18-59 anos). Foram coletados dados de 150 prontuários, que posteriormente foram computados e analisados em softwares adequados. RESULTADOS: Foram detectados 145 pacientes registrados no HIPERDIA dentro da faixa etária analisada, destes 56\% (82) possuíam HAS, destes, 70,7\% eram mulheres (58). Na distribuição por idade, avaliou-se maior prevalência na faixa etaria entre 50-59 anos com 53,7\% (44). Com relação aos indicadores de risco, como alcoolismo, tabagismo e IMC $>25 \mathrm{~kg} / \mathrm{m}^{2}$ foram detectadas respectivamente frequências de 20,7\% (17) 13,4\% (11), e 43,9\% (36). CONCLUSÃO: A maioria dos pacientes registrados no HIPERDIA possuíam HAS, que é mais prevalente conforme o envelhecimento dos pacientes. Com relaçã̃o aos fatores de risco, foi detectado maior prevalência em pessoas com algum grau de obesidade e subnotificação com relação ao tabagismo e o alcoolismo, visto que $31 \%$ dos prontuários não possuíam estas informações preenchidas.

Palavras-chave: Hipertensão; Doenças Cardiovasculares; Perfil Epidemiológico; População; Doença Crônica.

\begin{abstract}
INTRODUCTION: Systemic Arterial Hypertension (SAH) causes several cardiovascular comorbidities, which are related to worse prognosis and increased risk of death. Programs such as HIPERDIA have been implemented in Brazil, which perform the screening, monitoring and treatment of patients with SAH and Diabetes Mellitus, in order to reduce their impact on public health. So, it is necessary to know the profile of the patients registered in this program. OBJECTIVES: To identify the prevalence of SAH in adults in the ESF Mucajá, creating an epidemiological profile of these hypertensive patients. METHODS: Retrospective study, in which data such as sex, age, BMI, smoking and alcoholism were collected from medical records of ESF Mucajá patients who were in the determined age range (1859 years). Data were collected from 150 medical records, which were later computed and analyzed using appropriate software. RESULTS: 145 patients registered in HIPERDIA were detected within the analyzed age range, of these $56 \%$ (82) had SAH, of these, $70.7 \%$ were women (58). In the age distribution, a higher prevalence in the age group between 50-59 years was evaluated, with 53.7\% (44). Regarding risk indicators, such as alcoholism, smoking and BMI $>25 \mathrm{~kg} / \mathrm{m}^{2}$, frequencies of $20.7 \%$ (17), 13.4\% (11), and $43.9 \%$ (36) were detected respectively. CONCLUSION: Most patients registered with HIPERDIA had SAH, which is more prevalent as patients age. About risk factors, a higher prevalence was detected in people with some degree of obesity and underreporting in relation to smoking and alcoholism, since $31 \%$ of the medical records did not have this information filled out.
\end{abstract}

Keywords: Hypertension; Cardiovascular Diseases; Epidemiological Profile; Population; Chronic Disease.

Autor de correspondência

1- Acadêmicos do Curso de Medicina da Universidade do Estado do Pará (UEPA)

2- Acadêmica do Curso de Fisioterapia da Universidade do Estado do Pará (UEPA)

3- Acadêmicos do Curso de Fisioterapia da Faculdade de Ensino Paraense (FAPEN)

4- Acadêmico do Curso de Enfermagem da Universidade da Amazõnia (UNAMA)

5- Professora do Curso de Medicina da Universidade do Estado do Pará (UEPA)

6- FisioterapeutaProfessora do Curso de Medicina da Universidade do Estado do Pará (UEPA); Dra em Ciências da Reabilitação (UNINOVE)

Eduardo Augusto Silva Monteiro

Email: eduardo.monteiro@aluno.uepa.br

DOI: $\underline{10.36692 / v 13 n 1-36}$ 


\section{INTRODUÇÃO}

O advento da modernidade, além de trazer indeléveis benefícios para a sociedade atual, trouxe consigo mudanças, sobretudo, nos hábitos de vida praticados pelas pessoas que, do ponto de vista médico, não são saudáveis. Nessa perspectiva, a exposição a fatores de risco comportamentais, como o consumo de drogas, alimentação inadequada, falta de exercícios físicos, aumenta os riscos de desenvolvimento de doenças crônicas não transmissíveis, como a hipertensão $\operatorname{arterial}^{(1,2)}$. Tal doença, caracterizada por um aumento dos níveis pressóricos sobre as artérias, não raramente se associa a distúrbios metabólicos, alterações fisiológicas ou morfológicas nos órgãos afetados ${ }^{(3,4)}$

Responsável por cerca de 9,4 milhões de mortes, assim como a perda de 162 milhões de anos de vida, a Hipertensão Arterial Sistêmica (HAS) é responsável por metade das cardiopatias, dos acidentes vasculares cerebrais e das insuficiências cardíacas ${ }^{(5)}$. Essa enfermidade caracteriza-se, atualmente, como uma epidemia de saúde pública, cuja prevalência aumenta concomitantemente ao envelhecimento da população, atualmente, 4 em cada 10 adultos acima de 25 anos tem hipertensão ${ }^{(6,7,8)}$.

Doenças cardiovasculares, cujo principal fator de risco é a hipertensão arterial sistêmica, têm atuado como protagonistas nas causas de morte no Brasil, em 2016 tomaram-se cerca de $30 \%$ dos óbitos totais, sendo que, destes, cerca de $75 \%$ estavam associadas a doenças isquêmicas do coração, doenças cerebrovasculares e, ainda, doenças hipertensivas ${ }^{(9,10)}$. Nesse sentido, medidas de prevenção primária, a exemplo de promoção à saúde, como intervenções, ações educacionais e aconselhamentos atuam com importância ímpar ao impedir ou mesmo retardar o desenvolvimento destas doenças ${ }^{(11)}$.

Destacam-se como fatores de risco para a HAS os hábitos de vida insalubres, como os relacionados à alimentação com excesso de Sódio, a baixa ingestão de água e a inatividade física. Entretanto, não devem ser subestimados aspectos como idade, histórico familiar e genético, etnia e sexo, sendo que, ainda assim, o estilo de vida saudável é capaz de diminuir os riscos de desenvolvimento da hipertensão $\mathrm{O}^{(12,13,14)}$.

A presença desses fatores de risco e distúrbios metabólicos e fisiológicos, faz com que a HAS seja intimamente ligada com a morbidade e aparecimento de complicações, sobretudo, cardiovasculares ${ }^{(15)}$. Nesse viés, tornase extremamente necessário a individualização no atendimento, a fim de executar um bom diagnóstico e assumir uma correta decisão terapêutica ${ }^{(16)}$.

As complicações provenientes da HAS fazem com que os pacientes que apresentam tal quadro clínico procurem cuidados médicos que, na maioria dos casos, são de alto custo, tais como: constante uso de medicamentos, uma vez que a doença é crônica; exames complementares periódicos e afins ${ }^{(17)}$.

Dentre os mecanismos elaborados para responder às elevadas taxas de internações e na tentativa de alcançar a assistência e intervenção adequada na atenção primária, destaca-se o Plano de Reorganização da Atenção à Hipertensão Arterial (HA) e ao Diabetes Mellitus (DM), 
caracterizado por encontros mensais com estratégias pedagógicas, estímulos à pratica de atividades físicas, consultas com profissionais da saúde e entrega de medicamentos ${ }^{(18)}$. Neste contexto, o Sistema de Cadastramento e Acompanhamento de Hipertensos e Diabéticos (HIPERDIA), originado em 2012, cadastra e acompanha os pacientes portadores de HAS e ou $\mathrm{DM}$, além de oferecer dados para os responsáveis em saúde e administradores das Secretarias Municipais, Estaduais e Ministério da Saúde ${ }^{(19,20)}$ Embora exista um forte comprometimento de grande parte da equipe do NASF ao atuar no programa HIPERDIA, com o esforço de efetivação da adesão dos utilizadores do mesmo e afirmação de fortes vínculos destes com os profissionais, persistem brechas no processo de trabalho dos dirigentes ${ }^{(21)}$. Como observa-se no não comprometimento de todo o pessoal no trabalho, em especial os profissionais médicos, além da indisponibilidade de recursos de qualidade e em quantidade, sejam físicos ou materiais, para a concretização da potencialidade deste serviço e real adesão dos usuários ${ }^{(22)}$.

Os pacientes que apresentam um quadro hipertensivo caracterizam-se por ter uma pressão sistólica e diastólica igual ou maior a 140/90 $\mathrm{mmHg}$, sendo que 120/80 mmHg é considerada normal e 130/85 mmHg seria ótima, o que faz com que o coração tenha que trabalhar de maneira diferente para suprir as necessidades sanguíneas do organismo. Portanto, observa-se que a medida da pressão arterial é essencial para o diagnóstico de HAS, devendo ser aferida por meio de métodos e condições apropriados ${ }^{(23)}$.

A aferição da pressão arterial é um método que deve ser realizado constantemente, ainda que se obtenham resultados iniciais padrões para normalidade, visto que pode vir a provocar sintomas somente em fases demasiadamente avançadas ou quando eleva-se de maneira repentina, ademais, proporciona conhecimentos acerca do desempenho cardiovascular dos indivíduos ${ }^{(24)}$.

Perante este contexto, faz-se necessário a realização de estudos para analisar o perfil epidemiológico da Hipertensão Arterial Sistêmica, visto a prevalência da doença na população e agravos que a enfermidade pode vir a gerar. Visando-se obter melhorias e a implementação de políticas públicas de saúde capazes de combater a HAS oferecidas ao público.

\section{MÉTODOS}

O estudo foi realizado a partir do seguimento das normas éticas estabelecidas pelo Código de Nuremberg e pela Declaração de Helsinque, respeitadas as Normas de Pesquisa Envolvendo Seres Humanos (Res. CNS 466/12) do Conselho Nacional de Saúde e teve início após a submissão e aprovação do projeto pelo Comitê de Ética em Pesquisa da Universidade do Estado do Pará sob número 3.215.340 de 22 de março de 2019, e pela Estratégia Saúde da Família Mucajá. A pesquisa foi conduzida a partir da análise de prontuários dos pacientes da Estratégia Saúde da Família Mucajá, com consentimento dos pacientes a partir do Termo de Consentimento Livre e Esclarecido, ou quando esses não forem possíveis de serem encontrados, por meio do Termo de Consentimento de Uso de Dados. 
Foi realizado um estudo transversal, descritivo, e retrospectivo a partir da análise e levantamento de dados dos prontuários de pacientes da ESF Mucajá, especificamente da Equipe de Saúde da Família 1 dessa unidade. O estudo não possuiu intervenção clínica sobre os participantes. Compuseram a casuística jovens adultos, na faixa etária de 18 a 59 anos, que se enquadraram nos critérios de inclusão e exclusão apresentados. O período determinado para a análise dessa prevalência é de casos ocorridos a partir do ano de 2013 a 2018.

A pesquisa foi realizada na Estratégia Saúde da Família Mucajá, localizada na Passagem Mucajá, sem número, entre Avenida Pedro Álvares Cabral e Avenida Senador Lemos, bairro da Sacramenta, situado no município de Belém/ PA.

Foram avaliados uma amostra $\mathrm{n}$ de 151 prontuários de jovens adultos, número este calculado segundo a fórmula do cálculo do espaço amostral, na qual o índice de erro amostral e tolerável foi de $5 \%$, com índice de confiança de $95 \%$ em uma população heterogênea, a partir de uma população $\mathrm{N}$ de 246 jovens adultos já diagnosticados com HAS pela Equipe de Saúde da Família 1, que possuam prontuário na ESF Mucajá.

As informações foram obtidas após a aprovação no CEP UEPA a partir do levantamento de dados dos prontuários da ESF Mucajá, após a autorização realizada através da assinatura do TCUD elaborado pela equipe de pesquisa e validados pela orientadora, após a autorização pela coordenação da ESF Mucajá.

Foram coletados os seguintes dados conforme o protocolo de coleta para o estudo: idade, sexo, presença ou ausência de HAS, além de possíveis fatores de risco do paciente, se estes dados estiverem disponíveis. Os dados obtidos foram de natureza analítica e descritiva, sendo obtidos frequências absolutas e relativas de pacientes com HAS.

Após a coleta dos dados dos prontuários da amostra, os dados coletados foram então utilizados nos softwares Microsoft Excel 2016 e Microsoft Word 2016 que os quantificaram, transformando-os em gráficos e tabelas, sendo feito o cálculo da frequência absoluta e relativa de pacientes com HAS, além do cálculo do desvio padrão.

Os pacientes também foram divididos em subgrupos baseados em sua faixa etária, de maneira a facilitar a análise e identificar possíveis prevalências maiores em determinado grupo, etário: subgrupo 1, de 18 a 29 anos; subgrupo 2, de 30 a 39 anos; subgrupo 3, de 40 a 49 anos; subgrupo 4, de 49 a 59 anos. Também foi feita a análise da prevalência com relação ao sexo dos indivíduos estudados, buscando-se averiguar se existe diferença significativa entre suas frequências.

Foram incluídos nesse estudo pacientes atendidos pela Equipe de Saúde da Família 1da ESF Mucajá, entre 18 a 59 anos, homens e mulheres de qualquer renda, que tenham registrado hipertensão arterial comprovada, pressão sistólica maior que $140 \mathrm{mmHg}$ e 
pressão diastólica maior que $90 \mathrm{mmHg}$, ou pressão normotensa com uso de medicamentos hipotensores, que tenham ocorrido nos últimos 5 anos, cadastrados na ESF Mucajá.

Foram excluídos da pesquisa casos que não tenham ocorrido dentro da área de abrangência da Equipe de Saúde da Família 1, da ESF Mucajá, ou prontuários cujos dados estejam inespecíficos de maneira que seja impossibilitada a análise.

\section{RESULTADOS}

Os prontuários dos quais os dados foram coletados pertenciam a pasta do HIPERDIA da equipe X da USF Mucajá, pasta na qual são separados apenas os prontuários que possuem as doenças rastreadas pelo programa (Diabetes
Mellitus e HAS), foi feita uma pré-seleção nos prontuários coletados afim de selecionar os prontuários que se encaixavam na idade determinada no método, entre 18-60 anos. Assim, foram analisados 150 prontuários.

Destes 150 prontuários, 5 prontuários não se encaixavam nos critérios de inclusão de idade e foram excluídos da análise. Sobrando 145 prontuários, .63destes foram excluídos da análise do perfil epidemiológico porque eram pacientes registrados no HIPERDIA, mas que possuíam somente Diabetes Mellitus, logo, não possuindo HAS. Restaram então 82 prontuários de pacientes com HAS.

Os resultados da análise estão expostos nas seguintes tabelas e gráficos:

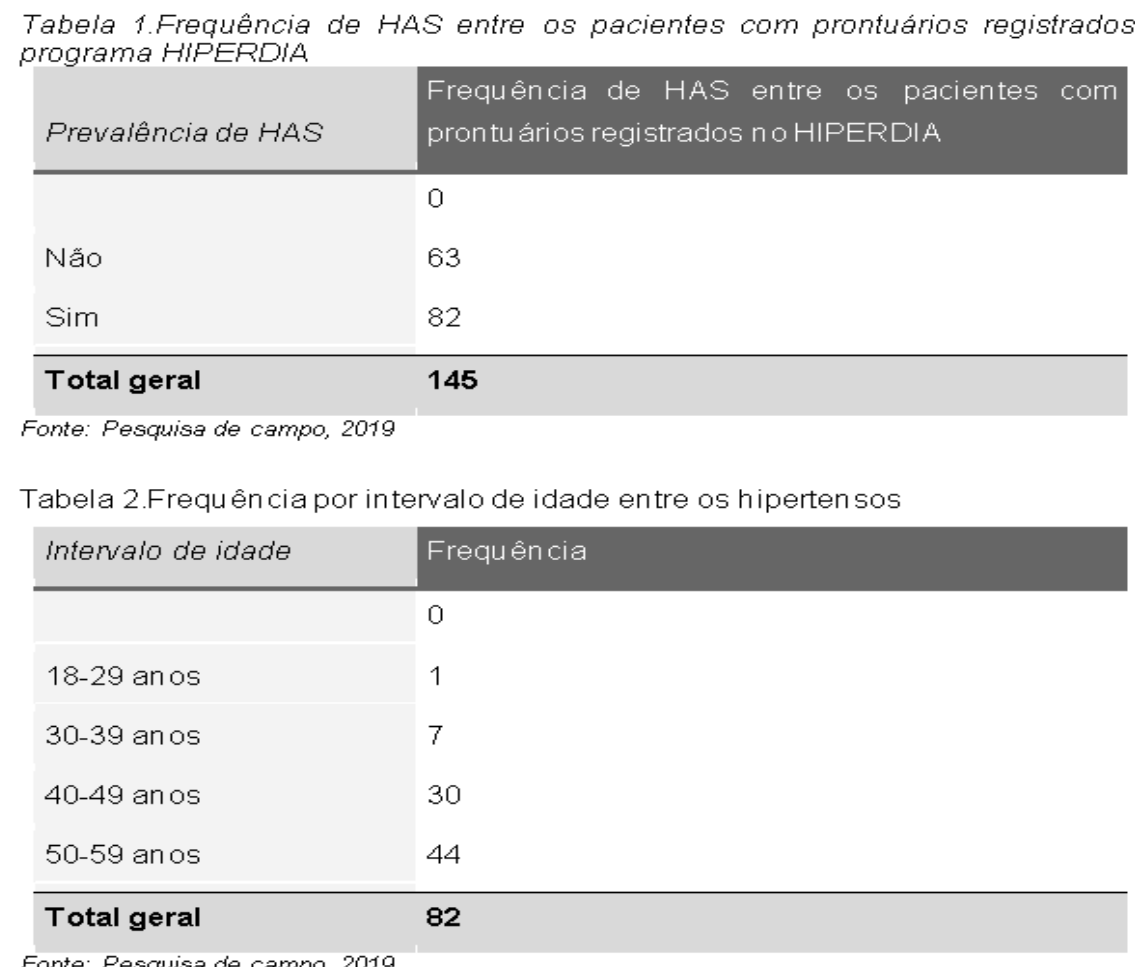




\begin{tabular}{|c|c|c|}
\hline Sexo & $\begin{array}{l}\text { idade } \\
\text { Subgrupo }\end{array}$ & Frequência \\
\hline & & 0 \\
\hline Total & & 0 \\
\hline \multirow[t]{3}{*}{ Feminino } & 30-39 anos & 3 \\
\hline & $40-49$ anos & 20 \\
\hline & $50-59$ anos & 35 \\
\hline \multicolumn{3}{|l|}{ Feminino } \\
\hline \multirow[t]{4}{*}{ Masculino } & $18-29$ anos & 1 \\
\hline & 30-39 anos & 4 \\
\hline & $40-49$ anos & 10 \\
\hline & $50-59$ anos & 9 \\
\hline \multicolumn{3}{|l|}{ Masculino } \\
\hline Total & & 24 \\
\hline Total geral & & 82 \\
\hline
\end{tabular}

Tabela 4.Frequência de alcoolismo entre os hipertensos

\begin{tabular}{ll} 
Alcoolismo & Frequência \\
\hline & 0 \\
Nẫo & 39 \\
Não possivel & \\
identificar & 26 \\
Sim & 17 \\
\hline Total geral & $\mathbf{8 2}$ \\
\hline Fonte: Pesquisadecamo, 2019
\end{tabular}

Fonte: Pesquisa de campo, 2019

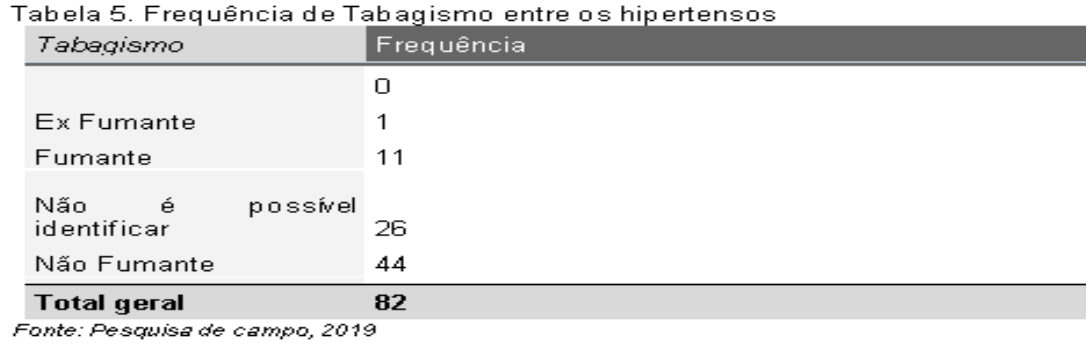

Tabela 6 . Frequência do IMC relacionado ao sexo entre os hipertensos

\begin{tabular}{|c|c|c|}
\hline MC & Sexo & Frequência \\
\hline & & 0 \\
\hline Total & & 0 \\
\hline \multirow[t]{2}{*}{ Maior que $25 \mathrm{~kg} / \mathrm{m}$ cúbico } & Feminino & 22 \\
\hline & Masculin & 14 \\
\hline $\begin{array}{l}\text { Maior que } 25 \mathrm{~kg} / \mathrm{m} \text { cúbico } \\
\text { Total }\end{array}$ & & 36 \\
\hline \multirow[t]{2}{*}{ Menor que $25 \mathrm{Kq} / \mathrm{m}$ cúbico } & Feminino & 26 \\
\hline & $\begin{array}{l}\text { Masculin } \\
0\end{array}$ & 9 \\
\hline $\begin{array}{l}\text { Menor que } 25 \mathrm{~kg} / \mathrm{m} \text { cúbico } \\
\text { Total }\end{array}$ & & 35 \\
\hline \multirow[t]{2}{*}{ Não é possível identificar } & Feminino & 10 \\
\hline & $\begin{array}{l}\text { Masculin } \\
0\end{array}$ & 1 \\
\hline $\begin{array}{l}\text { Não é possível identificar } \\
\text { Total }\end{array}$ & & 11 \\
\hline Total geral & & 82 \\
\hline
\end{tabular}




\section{DISCUSSÃO}

A Hipertensão Arterial apresenta elevada prevalência na população brasileira e em âmbito mundial. De acordo com estudos, o estilo de vida contemporâneo, que é caracterizado por consumo crescente de alimentos não saudáveis e pouca prática de exercício físico, coopera para o aumento da prevalência da doença na população ${ }^{(25)}$. Além disso, tem- se constatado também, como consequência do atual cenário mundial, um aumento significativo da HAS em indivíduos cada vez mais jovens, tanto em países em desenvolvimento quanto em países desenvolvidos ${ }^{(26)}$.

De acordo com estudos, no Estado do Rio de Janeiro, a prevalência de HAS encontrada na população adulta foi de $23,9 \%{ }^{(27)}$. Porém, a vigente pesquisa constatou que em uma amostra de 145 prontuários, 82 indivíduos tinham sido diagnosticados com a doença. Dessa maneira, a prevalência de Hipertensão Arterial Sistêmica $(56,55 \%)$ constatada foi maior do que a esperada na ESF Mucajá.

Em relação à associação da HAS com a idade, foi verificado que o número de indivíduos acometidos aumentou com a idade. O maior número de casos ocorreu em indivíduos na faixa etária de 50 a 59 anos (44; 53,65\%), seguido por pessoas com idade entre 40 a 49 anos (30; $36,58 \%), 30$ a 39 (7; 8,53\%), 18 a 29 (1; 1,21\%). Esse fato está de acordo com relatos das literaturas e pode ser explicado por mudanças típicas do envelhecimento, como o enrijecimento das artérias e aumento da resistência vascular periférica ${ }^{(28)}$.
Já ao relacionar a hipertensão arterial e o índice de Massa Corporal (IMC) obteve-se que 43,9\% dos pesquisados, logo, a maioria, possui $\mathrm{IMC}>25 \mathrm{Kg} / \mathrm{m}^{2}$, sendo que, de 36 pessoas, 22 são do sexo feminino. Entrando assim em concordância com um estudo realizado em São Paulo(29), o qual demonstrou que o IMC em faixas elevadas aumenta em 2,2 vezes as chances de Hipertensão Arterial Sistêmica.

Entretanto $42,7 \%$ dos pesquisados foram classificados com o IMC menor que $25 \mathrm{Kg} / \mathrm{m} 3$. Portanto não se pode estabelecer uma relação direta com sobrepeso ou obesidade com o aumento dos níveis pressóricos, apesar de saberse que ambos constituem um problema de saúde públicaimportante na sociedade e estão associados a diversas doenças crônico-degenerativas, sendo a Hipertensão Arterial Sistêmica uma delas ${ }^{(30,31)}$.

Quanto ao consumo de bebida alcoólica entre os hipertensos, encontra-se uma grande dificuldade para quantificar os padrões de consumo, uma vez que, nessa população, ainda há uma grande resistência à participação em protocolos de pesquisa. Dentre os pesquisados, 20,73\% dos hipertensos fazem consumo de bebida alcoólica de maneira social (em dose baixa), podendo-se, assim, inferir que não há uma relação direta entre a ingestão de álcool e a elevação sustentada dos níveis pressóricos. Entretanto, existe o efeito dose-dependente, pois o consumo crônico e elevado de bebidas alcoólicas aumenta a PA de forma consistente ${ }^{(3)}$.

No que diz respeito ao tabagismo, foi constatado que $13,41 \%$ dos hipertensos são 
fumantes, e apenas 1,21\% relatou ser ex-fumante. Apesar de pouco, numericamente, tal dado é alarmante, pois o cigarro exerce uma relação direta no aumento da PA e suas complicações. Essa informação foi confirmada pelo estudo INTERHEART ${ }^{(32)}$, que analisou os fatores de risco associados a uma das principais complicações da hipertensão arterial: alto risco cardiovascular. Tal estudo foi baseado na análise dos fatores de risco, em 52 países, associados ao infarto agudo do miocárdio. Pôde-se observar que fumar de um a cinco cigarros/dia resultou em um aumento do risco relativo de $38 \%$, enquanto acima de quarenta cigarros/dia, tal risco pode ser multiplicado 9,16 - Também foi comprovado que uma redução no tabagismo pode ser correlacionada com uma acentuada diminuição no risco cardiovascular.

A presente pesquisa apresenta como relevante limitação as dificuldades de acesso às informações dos prontuários de pacientes atendidos na unidade. Destaca-se que muitos prontuários foram apontados como em constante processo de atualização, e, visto que encontram-se em formato escrito em detrimento do digital, poderia ser que não estivesse sendo utilizado os dados mais atuais, representando menos precisamente a população estudada. O prontuário escrito contribuiu ainda para a ocorrência de dificuldades de entendimentos gramaticais pelos pesquisadores. Outro fator relevante de ser destacado é o fato de que muitas informações, a exemplo das que identificam os pacientes como diabético, etilista ou tabagista, não constavam como preenchidas em diversos registros, dificultando a análise sistemática e qualitativa da pesquisa em questão.

\section{CONCLUSÃO}

A partir dos dados coletados, não é possível comparar o número de casos de HAS e estabelecer uma porcentagem de adultos hipertensos na Unidade de Saúde da Família Mucajá, visto que os prontuários dos pacientes hipertensos eram separados em uma pasta separada do programa HIPERDIA. Além disso, as ACSs da USF não contabilizavam os atendimentos em demanda de adultos, por se tratar de uma quantidade exorbitante de atendimentos diários.

Entretanto, foi levantado que entre os pacientes com prontuários registrados no programa HIPERDIA da unidade (que envolve pacientes acometidos por a HAS ou Diabetes Mellitus), os pacientes adultos hipertensos representaram a maioria (82 pacientes de uma amostra de 145).

A partir da análise do perfil epidemiológico dos adultos hipertensos analisados conclui-se que a sua maioria são de adultos entre 40-59 anos (74 de uma amostra de 82). A maioria destes adultos são do sexo feminino (58 de uma amostra de 82).

Ao relacionar os fatores tabagismo, alcoolismo e IMC, foi possível observar que parte significativa dos prontuários, cerca de $26 \%$ no caso dos fatores tabagismo e alcoolismo e cerca de $11 \%$ no fator IMC, não apresentava essas informações disponíveis, o que indica uma carência no preenchimento dos dados dos prontuários por parte de alguns funcionários da USF. 
Portanto, o presente estudo foi capaz de determinar a maior prevalência de hipertensão entre adultos mais velhos, além de levantar o perfil epidemiológico dos adultos hipertensos da unidade de saúde analisada. Entretanto, faz-se necessário a realização de estudos mais amplos e aprofundados afim de determinar o indicador epidemiológico correlacionando o volume de adultos atendidos com o número de adultos hipertensos da Unidade de Saúde da Família Mucajá.

\section{REFERÊNCIAS}

1. Malta DC, Andrade SSCDA, Oliveira TP, Moura LD, Prado RRD, Souza MDFMD. Probabilidade de morte prematura por doenças crônicas não transmissíveis, brasil e regiões, projeções para 2025. Rev bras epidemiol. 2019;22:1-13.

2. Hortencio MNS, Silva JKS, Zonta MA, Melo CPA, França CN. Effects of physical exercise on cardiovascular risk factors in hypertensive older adults. Revista Brasileira em Promoção da Saúde. 2018;31(2):19.

3. Sociedade brasileira de cardiologia (SBC). VII Diretriz brasileira de hipertensão. Arquivos Brasileiros de Cardiologia. 2016; 3(1):1-3.

4. Engela MHT, Rodarte AC, Rotondaro Júnior A, Seixas CT, Viegas SMDF, Lanza FM. Uso das tecnologias em saúde na atenção básica às pessoas em condições de hipertensão arterial sistêmica. J. res.: fundam. care. online. 2018;10(1):75-84.

5. Malta DC, Gonçalves RPF, Machado ÍE, Freitas MIDF, Azeredo C, Szwarcwald CL. Prevalência da hipertensão arterial segundo diferentes critérios diagnósticos, Pesquisa Nacional de Saúde. Rev Bras Epidemiol. 2018;21:1-15.

6. Campbell NRC, Lackland DT, Niebylski ML. High Blood Pressure: Why prevention and control are urgent and important - A 2014 fact sheet from the world hypertension league and international society of hypertension. The Journal of Clinical Hypertension. 2014;16(8):551-553.

7. Agostini CM, Rodrigues VS, Guimarães, AC, Damázio LCM, Vasconcelos NN. Análise do desempenho motor e do equilíbrio corporal de idosos ativos com hipertensão arterial e diabetes tipo 2. Rev. Aten. Saúde. 2018;16(55):29-35.

8. Sousa ALL, Batista SR, Sousa AC, Pacheco JAS, Vitorino PVO, Pagotto V. Prevalência, Tratamento e Controle da Hipertensão Arterial em Idosos de uma Capital Brasileira. Arq Bras Cardiol. 2019; 112(3):271278.
9. Departamento de Informática do Sus. Informações de Saúde, Epidemiológicas e Morbidade: banco de dados (Datasus). Disponível em: <http://www2.datasus.gov.br/DATASUS/index. php? area $=0205>$ Acesso em 04 de novembro de 2018.

10. Tanaka OY, et al. Arterial hypertension as a tracer for the evaluation of access to health care. Ciência \& Saúde Coletiva, 24(3):963-972, 2019.

11. Almeida LM, Campos KFC, Randow R, Almeida VG. Estratégias e desafios da gestão da atenção primária à saúde no controle e prevenção da obesidade. Revista Eletrônica Gestão e Saúde. 2017;8(1):114-139.

12. National Heart, Lung and Blood Institute (NHLBI). High Blood Pressure. Disponível em < http:/ / www. nhlbi.nih.gov/health/healthtopics/topics/hbp/ atrisk $>$ Acesso em 03 novembro 2018.

13. Firmo JOA, Mambrini JVDM, Peixoto SV, Loyola Filho AID, Souza Junior PRBD, Andrade FBD, et al. Controle da hipertensão arterial entre adultos mais velhos: ELSI-Brasil. Rev Saude Publica. 2018;52: 1-11.

14. Silva MGC, Domingos TS, Caramaschi S. Hipertensao arterial e cuidados com a saude: concepções de homens e mulheres. Psicologia, saúde \& doenças. 2018;19(2):435-452.

15. Soares FC, et al. Prevalência de hipertensão arterial e diabetes mellitus em portadores de doença renal crônica em tratamento conservador do serviço ubaense de nefrologia. Revista Científica Fagoc Saúde Volume II - 2017

16. Ferreira LR, Artmann E. Discursos sobre humanização: profissionais e usuários em uma instituição complexa de saúde. Ciência \& Saúde Coletiva. 2018;23(5):1437-1450.

17. Nilson EAF, Andrade RCS, Brito DA, Oliveira ML. Custos atribuíveis a obesidade, hipertensão e diabetes no Sistema Único de Saúde. Rev Panam Salud Publica. 2020;44:e32.

18. Pessanha CJS. O contexto da saúde brasileira e o enfrentamento ao avanço do diabetes mellitus no brasil: a implantação do plano de reorganização da atenção à hipertensão arterial e ao diabetes mellitus (prahadm). Rev.Mundo Livre. 2020; 6(2):283-305.

19. Silva JVM, Mantovani MF, Kalinke LP, Ulbrich EM. Hypertencion and diabetes mellitus program evaluation on user's view. Revista Brasil Enfermagem. 2015;68(4): 626-632.

20. Nicolau S, Batista KJD, Moura A, Simas J. Práticas de educação em saúde realizadas por enfermeiros para pacientes do programa hiperdia. J Manag Prim Health Care. 2018;9:e9.

21. Sousa NA, Lima JS, Teixeira TC, Linhares CB, Montes JVL, Marques JVS. Fatores de risco e complicações em diabéticos/hipertensos cadastrados no hiperdia. Sanare. 2019;18(1):31-39.

22. Santos AL, Silva EME, Marcon SS. Assistência às pessoas com diabetes no hiperdia: potencialidades e limites na perspectiva de enfermeiros. Texto Contexto Enfermagem. 2018: 27(1).

23. Francisco PMSB, Segri NJ, Borim FSA, Malta DC. Prevalência simultânea de hipertensão e diabetes em idosos brasileiros: desigualdades individuais e contextuais. Ciência \& Saúde Coletiva. 2018;23(11):38293840 .

24. Ministério da Saúde (Brasil). Secretaria de Vigilância em Saúde. Departamento de Vigilância de 
Doenças e Agravos não Transmissíveis e Promoção da Saúde. Uma Análise da Situação de Saúde e das Causas externas. Brasília, DF; 2015. Disponível em: http://bvsms.saude.gov.br/bvs/publicacoes/saude_ brasil_2014_analise_situacao.pdf

25. Zangirolani LTO, Assumpção D, Medeiros MAT, Barros MBA. Hipertensão arterial autorreferida em adultos residentes em Campinas, São Paulo, Brasil: prevalência, fatores associados e práticas de controle em estudo de base populacional. Ciência \& Saúde Coletiva. 2018; 23(4):1221-1232.

26. Moura IH, Vieira EES, Silva GRF, Carvalho RBN, Silva ARV. Prevalência de hipertensão arterial e seus fatores de risco em adolescentes. Acta Paulista de Enfermagem. 2015;28(1):81-86.

27. Neto VGC, Monteiro ER, Silva ALS, Faria FO, Palma A. Prevalência de Hipertensão Arterial Sistêmica em Estudantes de Educação Física de uma Universidade Pública no Rio de Janeiro. J Health Sci. 2017; 19(4): 241244.

28. Queiroz MG, et al. Arterial hypertension in the elderly -prevalent disease in this population: an integrative review. Braz. J. of Develop.,Curitiba, v.6, n.4,p. 22590-22598apr.2020.

29. Silveira EA, Vieira LL, Souza JD. Elevada prevalência de obesidade abdominal em idosos e associação com diabetes, hipertensão e doenças respiratórias. Ciência \& Saúde Coletiva, 23(3):903-912, 2018.

30. Ferreira APS, Szwarcwald CL, Damacela GN. Prevalência e fatores associados da obesidade na população brasileira: estudo com dados aferidos da Pesquisa Nacional de Saúde, 2013. REV BRAS EPIDEMIOL 2019; 22: E190024.

31. Martins APB. É preciso tratar a obesidade como um problema de saúde pública. (C) RAE | São Paulo | V. 58 | n. 3 | maio-jun 2018 | 337-341

32. Yusuf S, Hawken S, Ounpuu S, Dans T, Avezum A, Lanas F. et al . Effect of potentially modifiable risk factors associated with myocardial infarction in 52 countries (the INTERHEART study): case-control study. Lancet. 2004;364(9438):937-52

OBSERVAÇÃO: Os autores declaram não existir conflitos de interesse de qualquer natureza. 